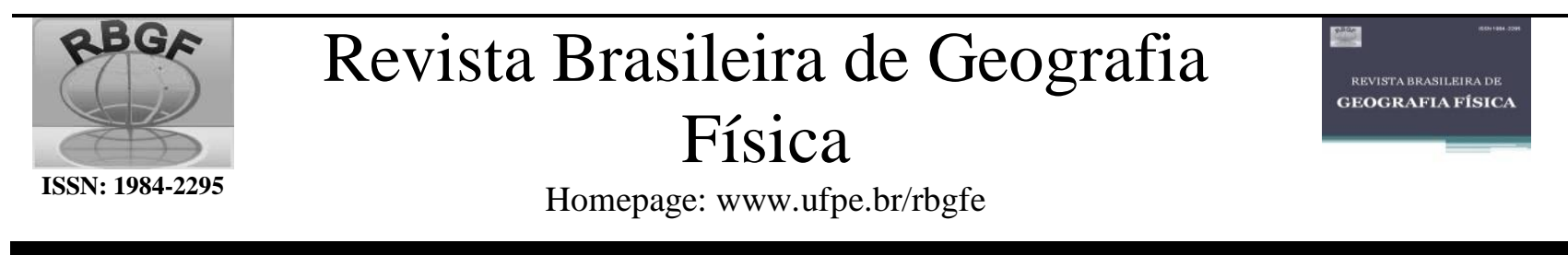

\title{
Estrutura de um Fragmento de Caatinga Infestado por Cryptostegia madagascariensis Bojer ex Decne ${ }^{1}$
}

\author{
Elaine Maia Barbosa², Oriel Herrera Bonilla³ , Eliseu Marlônio Pereira de Lucena ${ }^{4}$, Lívia Maria de Andrade \\ Araújo $^{5}$, Sara Regina da Silva de Oliveira ${ }^{6}$
}

\begin{abstract}
${ }^{1}$ Parte da pesquisa de Mestrado, executada pelo primeiro, orientada pelo segundo e coorientada pelo terceiro autor, no âmbito do Mestrado Acadêmico em Ciências Naturais na Universidade Estadual do Ceará, Fortaleza, CE.

${ }^{2}$ Mestre, Mestrado Acadêmico em Ciências Naturais, Universidade Estadual do Ceará, Campus Itaperi, CEP 60.714-903, Fortaleza, CE. E-mail: elainemaiab@gmail.com - autor correspondente.

${ }^{3,4}$ Doutor, Professor Associado do Curso de Ciências Biológicas e do Mestrado Acadêmico em Ciências Naturais, Universidade Estadual do Ceará, Campus Itaperi, CEP 60.714-903, Fortaleza, CE. E-mail: oriel.herrera@uece.br e eliseu.lucena@uece.br.

${ }^{5}$ Discente do curso de Física e aluna de iniciação científica do Laboratório de Ecologia, Universidade Estadual do Ceará, Campus Itaperi, CEP 60.714903, Fortaleza, CE. E-mail: livia.andrade@aluno.uece.br.

${ }^{6}$ Discente do curso de Ciências Biológicas e aluna de iniciação científica do Laboratório de Ecologia, Universidade Estadual do Ceará, Campus Itaperi, CEP 60.714-903, Fortaleza, CE. E-mail: sara.regina@aluno.uece.br.
\end{abstract}

Artigo recebido em 27/05/2019 e aceito em 25/07/2019

\section{R E S U M O}

A espécie Cryptostegia madagascariensis Bojer ex Decne., natural de Madagascar, na África, após ser introduzida no Brasil vem proliferando-se rapidamente em extensas áreas no Nordeste brasileiro. Sua presença no bioma caatinga vem causando grande preocupação para as comunidades extrativistas, visto que essa invasora causa morte de espécies nativas de alto valor socioeconômico como a Copernicia prunifera (Mill) H. E. Moore., popularmente chamada de carnaúba. O presente trabalho objetivou avaliar a estrutura de um fragmento de caatinga infestado por C. madagascariensis. Próximo ao município de Pacatuba-CE uma área de carnaubal foi delimitada e para realizar uma avaliação da estrutura da comunidade invadida, foram utilizados cálculos que são referências em estudos fitossociológicos como, índice de valor de importância, índice de Shannon-Wiener (H'), índice de impacto ambiental de espécies exóticas (IIAE), além das densidades e frequências relativas e absolutas dos indivíduos. A elevada frequência e densidade relativa da invasora, o baixo H' $(1,86)$ e IIAE de 0,69 , demonstram como a estrutura e diversidade da comunidade está sendo afetada pela invasão. Concluímos que é urgente a intervenção e controle dessa invasora, uma vez que a vegetação nativa está sendo ameaçada.

Palavras-chave: Invasão, biodiversidade, carnaúba.

\section{Structure of a Fragment of Caatinga Infested by Cryptostegia madagascariensis Bojer ex Decne}

\begin{abstract}
A B S T R A C T
The species Cryptostegia madagascariensis Bojer ex Decne., natural from Madagascar, in Africa, after being introduced in Brazil, is pullulating quickly extensive areas of Brazilian Northeast. Its presence in the caatinga bioma is causing considerable preoccupation to the extractivist communities, since this invader is causing the death of native species with high socioeconomic value as the Copernicia prunifera (Mill) H. E. Moore., popularly known as carnaúba. The objective of the present study was to evaluate the structure of a fragment of caatinga infested by $C$. madagascariensis. Nearby the city of Pacatuba, CE, an area of carnauba forest was delimited to perform an evaluation of the invaded community structure. We utilized calculations that are reference in phytosociological studies as, importance value index, ShannonWiener Function (H'), environmental impact of alien species (IIAE), densities, relative and absolute frequencies of the subjects. The high frequency and relative density of the invasive species, the low H' (1.86) and 0.69 IIAE, demonstrate how the structure and diversity of the community is being affected by the invasion. We concluded that an intervention is urgent the intervention and control of this invasive species, since the native vegetation is being threatened.

Keywords: Invasion, biodiversity, carnaúba palm.

Introdução

As invasões biológicas vêm atraindo

gradativamente mais atenção devido aos impactos ambientais e econômicos que elas geram. Atualmente, essas invasões dificultam a
\end{abstract}


conservação da biodiversidade global (Maitre et al., 2015; Padalia et al., 2015), uma vez que espécies invasoras competem por recursos como, luz, nutrientes, água e espaço com espécies residentes (Souza et al., 2016; Nobis et al., 2018;).

Espécies invasoras vegetais causam elevados gastos financeiros em métodos utilizados na tentativa do controle. Essas, ainda quando não controladas, afetam a cadeia produtiva de uma região, devido aos distúrbios ecológicos causados, principalmente, em espécies utilizadas economicamente pela sociedade (Rumlevorá et al., 2016).

É necessário, para melhor gerenciamento dessas invasões, compreender quais características favoreceram o processo de invasão e quais ambientes tendem a ser mais susceptíveis à invasão do que outros, onde característica de cada espécie e do ambiente invadido devem ser levadas em consideração (Johnson e Léon, 2015).

Em diversos casos, a dificuldade no controle de espécies vegetais invasoras está relacionada com a semelhança de características fisiológicas que essas apresentam com as espécies nativas, onde, ainda, a carência de fatores limitantes como, alta capacidade de competição das espécies residentes, presença de predadores e doenças, contribuem para uma rápida proliferação e consequente dominância da invasora no ambiente (Maitre et al., 2015).

Ribeiro et al. (2017) mostra que algumas características reprodutivas e fisiológicas da espécie invasora podem facilitar sua proliferação. Exemplos de algumas das vantagens na competição pelo ambiente são, por exemplo, o elevado número de sementes por fruto, a alta percentagem de germinação, a longevidade de sementes, a regulação osmótica e a tolerância as mudanças ambientais (Skálová et al., 2015), ocasionando, assim, alterações no funcionamento e estrutura do ecossistema local (Gorgone-Barbosa et al., 2016, Nackley et al., 2017).

No Nordeste brasileiro é comum encontrar áreas que foram convertidas em pastagens e cultivos agrícolas serem abandonados (Ferreira et al., 2016). Esses ambientes degradados costumam ser regiões susceptíveis a invasões. Byun et al. (2017) explica que a diminuição da biodiversidade local diminui a resistência biótica à invasão, visto que, a diminuição da biodiversidade em uma área contribui para o estabelecimento de novas espécies exóticas.

No Brasil a fitofisionomia da caatinga vem enfrentando uma intensa degradação ambiental, onde o uso do solo e atividades agrícolas com introdução de plantas exóticas (Almeida et al., 2015) aceleram o processo de desertificação e homogeneização de espécies desse bioma.

Entre algumas das espécies vegetais invasoras que tem acelerado o processo de degradação do semiárido brasileiro temos, Calotropis procera, Prosopis juliflora, Nicotiana glauca (Fabricante et al., 2015), Sesbania virgata e Cryptostegia madagascariensis (Souza et al., 2016). Essas intensificam problemas ambientais, principalmente por competirem com espécies nativas e endêmicas que são de interesse econômico do semiárido (Nascimento et al., 2014).

A espécie $C$. madagascariensis pertence à família Apocynaceae e apresenta distribuição natural ao longo da região ocidental de Madagascar, na África. Crescendo em florestas secas, áreas agrícolas, savanas e em pastagens perturbadas, essa espécie apresenta porte arbustivo. No entanto em ambientes que invadem consegue desenvolver ramos laterais possibilitando que essa consiga utilizar plantas residentes como suporte atingindo, em alguns casos, até $15 \mathrm{~m}$ de altura (Sousa et al., 2016). Assim, C. madagascariensis ocasiona morte tanto das espécies que utilizam como suporte, como outras espécies que se encontrem em suas proximidades, devido ao sombreamento da área (deflação fotossintética) (Brito et al., 2015).

A germinação, floração e frutificação dessa invasora africana ocorrem durante o ano todo. No entanto a intensidade na produção de propágulos é recorrente em períodos chuvosos, assim como em solos com manchas úmidas (Sousa et al., 2017). A espécie ainda se caracteriza pela presença de látex presente em toda sua estrutura (Sousa et al., 2016).

Acredita-se que sua introdução tenha ocorrido no Brasil para fins ornamentais (Silva et al., 2018), devido a suas inflorescências de coloração lilás. No entanto essa espécie se adaptou ao semiárido brasileiro e, por apresentar rápida proliferação, a mesma vem causando modificações na estrutura das comunidades e homogeneização da biodiversidade em ambientes que colonizam (Brito et al., 2015).

Esses mesmos problemas também já foram registrados em outras regiões onde a espécie também é invasora como na Austrália, Marrocos, México e Havaí (Medeiros et al., 2018).

No Brasil os maiores índices de invasão de C. madagascariensis ocorrem nos estados do Nordeste, onde matas ciliares e planícies aluviais tem se mostrado regiões altamente invadidas por 
densos maciços dessa espécie. No Ceará essa invasora, também conhecida popularmente como unha-do-cão, localiza-se frequentemente nas margens de cursos d'água, intensificando a diminuição da vegetação da mata ciliar da caatinga (Cruz et al., 2016; Sousa et al., 2017).

Uma espécie endêmica que ocorre nas matas ciliares, principalmente, nos estados Piauí, Ceará e Rio Grande do Norte (Rocha et al., 2015), e que vem sofrendo intensa competição com $C$. madagascariensis é a palmeira Copernicia prunifera. Essa palmeira, pertencente à família Arecaceae, também conhecida popularmente como carnaúba, apresenta um alto valor socioeconômico para as comunidades extrativistas, sendo a diminuição dos carnaubais, devido a invasão de unha-do-cão no semiárido, uma grande preocupação social (Vieira et al., 2016).

A carnaúba é uma espécie vegetal de grande porte. Alcançando, aproximadamente, de 10 a $20 \mathrm{~m}$ de altura, seu estipe pode chegar a um diâmetro de 15 a $25 \mathrm{~cm}$. Suas folhas são palmadas, suas inflorescências são do tipo panícula e seus frutos do tipo baga com forma ovóide (Rocha et al., 2015). A emissão de folhas pode ser visualizada durante todo o ano. Essa emissão constante propicia a conservação da biodiversidade, uma vez que as áreas utilizadas pela atividade extrativistas são protegidas contra usos destrutivos como agropecuária e desmatamento (Costa e Gomes, 2016).

Pela grande diversidade de usos para o homem, $C$. prunifera também é conhecida como árvore da vida. Presente nas folhas o pó cerífero, matéria prima para a produção de cera, é responsável por movimentar grande parte da economia local. A cera das carnaúbas vem apresentando diversos fins nos mais variados campos da indústria (Costa e Gomes, 2016) como, aplicações na indústria cosmética, farmacêutica (Silva et al., 2018), alimentícia, eletrônica (Sousa et al., 2015). Pereira et al (2014) ainda apresenta que a cera apresenta alguns efeitos como antioxidante, anti-inflamatório e antifúngico.

Além da cera, as folhas de C. prunifera podem ser usadas na confecção artesanal, suas fibras podem ser usadas na obtenção de papel artesanal, seus frutos possibilitam alimentar animais do agronegócio, seus troncos por ótima resistência a pragas, são utilizados na construção de casas e suas raízes são usadas na produção de medicamentos para tratamentos de úlceras, erupções cutâneas e reumatismo (Sousa et al., 2015; Vieira et al., 2016).

Vale enfatizar que o impacto com espécies residentes no semiárido brasileiro não é o único problema econômico e ambiental relacionado com a presença de $C$. madagascariensis. Indícios de intoxicação alimentar causado pela espécie africana em animais do agronegócio já foram relatados, onde a morte desses animais gera perdas no capital financeiro, uma vez que a pecuária, assim como a atividade extrativista, é imprescindível para a movimentação da economia no país.

A observação do impacto de espécies invasoras na biodiversidade nativa tem contribuído para a conservação, recuperação e manejo dos ecossistemas, principalmente quando esses se encontram antropizados ou invadidos por espécies exóticas invasora. Nesse contexto, o presente trabalho objetivou avaliar a estrutura de um fragmento de caatinga infestado por Cryptostegia madagascariensis Bojer ex Decne.

\section{Material e métodos}

\section{Área de estudo}

O estudo foi realizado em um bosque de carnaúba, inserido no bioma caatinga, localizado próximo ao município de Pacatuba-CE (Figura 1) nas coordenadas geográficas $04^{\circ} 00^{\prime} 11^{\prime} \mathrm{S}$ e $38^{\circ}$ $35^{\prime} 58^{\prime}$ "WO. O estudo ocorreu durante o período seco, no mês de outubro. 

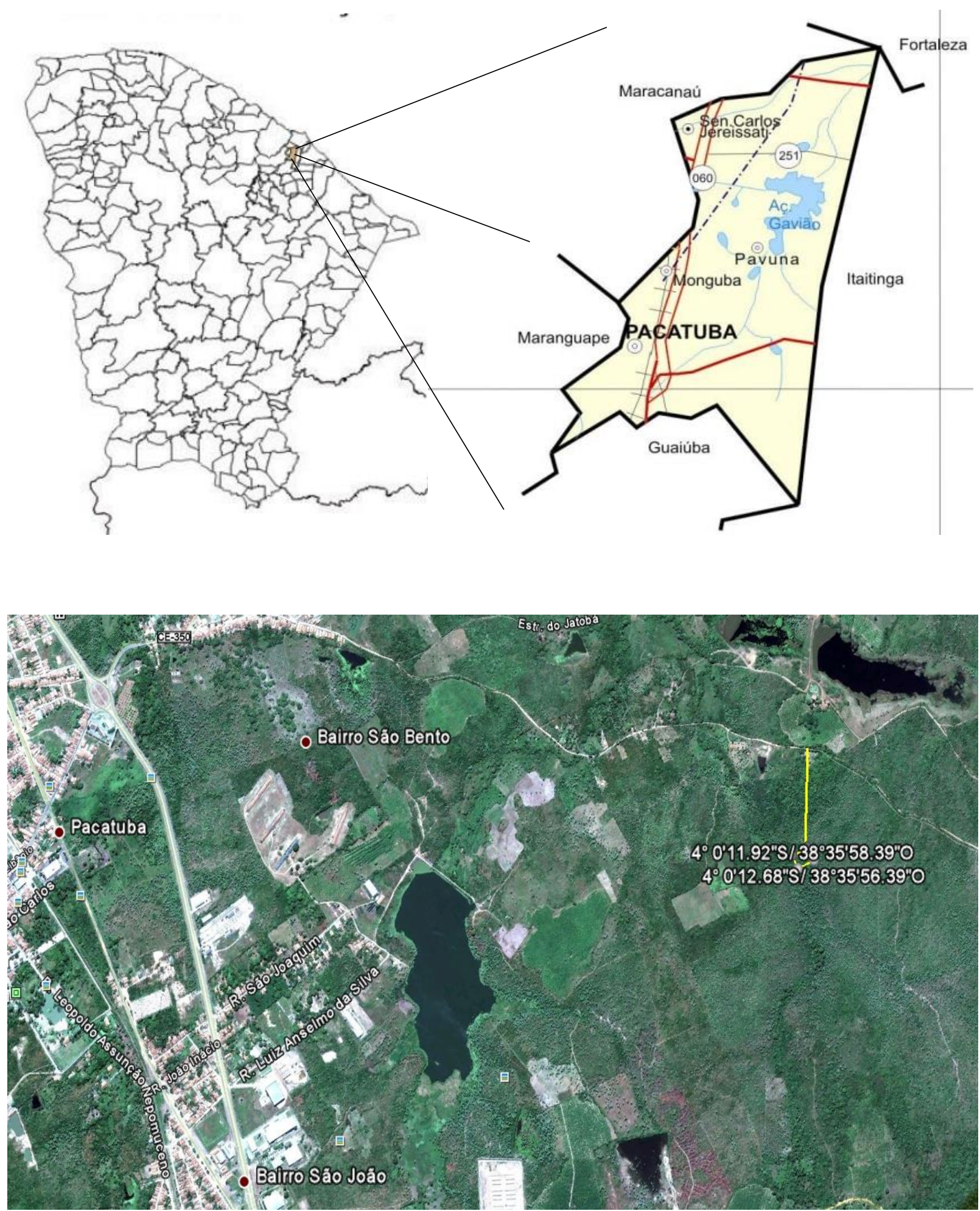

Figura 1. Localização geográfica do município de Pacatuba-CE e vista aérea da área experimental. Em amarelo rota de acesso para área experimental. Adaptado do IPECE (2009).

O clima da região é caracterizado como tropical quente úmido, apresentando verão chuvoso, entre os meses de janeiro a maio, com média pluviométrica de, aproximadamente, 1479,5 $\mathrm{mm}$, temperatura média anual entre 26 a $28^{\circ} \mathrm{C}$ e apresentando, predominantemente, solo podzólico (IPECE, 2017). Embora a região seja caracterizada semiárida sua proximidade de áreas montanhosas favorece uma elevada pluviometria, devido ao efeito orográfico (Silva et al., 2018).

A região delimitada para a realização do estudo era caracterizada por apresentar atividade extrativista, direcionada a retirada de folhas das carnaúbas, embora grandes extensões da região já 
se encontravam infestadas pela exótica invasora $C$. madagascariensis.

Amostragem da vegetação

A demarcação ocorreu pelo método de parcelas aleatórias, onde nenhuma tendência ou escolha de categorias de solo, vegetação ou estratificação foi utilizada. Foram delimitadas 81 subparcelas como unidades amostrais, apresentando dimensão de $9 \mathrm{~m}^{2}$ cada. Dentro de cada unidade todos os indivíduos lenhosos foram analisados com critério de inclusão de diâmetro à altura do peito (DAP), sendo esse, igual ou maior a $3 \mathrm{~cm}$ e com altura maior ou igual a $1 \mathrm{~m}$ (Felfili et al., 2011). O diâmetro dos indivíduos inventariados foi medido com fita métrica e a altura dos indivíduos foi estimada com auxílio de hastes de madeira com $1 \mathrm{~m}$ de altura (Felfili et al., 2011; Brand et al., 2015).

Todos os indivíduos presentes na área de estudo tiveram identificação botânica mediante a auxílio de mateiros e consultas na coleção botânica do Herbário Prisco Bezerra, localizado na Universidade Federal do Ceará.

Os parâmetros estruturais calculados foram os recomendados pela literatura especializada (Muller-Dombois e Ellemberg, 1974), tais como densidade absoluta (DA), densidade relativa (DR), frequência absoluta e frequência relativa (FA, FR), dominância (DoA, DoR) em seus valores absolutos e relativos e o índice de valor de importância de cada espécie encontrada na área estudada (IVI).

Também foi calculado o índice de diversidade de Shannon-Wiener (H') (Shannon e Weaver, 1949) e o impacto da espécie exótica Cryptostegia madagascariensis Bojer ex Decene sobre a comunidade invadida, por meio do índice de impacto ambiental de espécies exóticas (IIAE) (Reaser et al., 2007).

\section{Resultados e discussão}

No total, foram inventariados 430 indivíduos nas subparcelas pertencentes a 10 famílias, 16 gêneros e 17 espécies. A família de Cactaceae (duas espécies), Arecaceae (duas espécies) e Fabaceae (cinco espécies) foram as mais representadas na área estudada, sendo a família Fabaceae a de maior abundância também em outros trabalhos realizados no semiárido como Brand et al. (2015) e Costa e Mesquita (2016).

A lista florística levantada também incluiu espécies nativas registradas em outros estudos desenvolvidos na vegetação caatinga como Júnior et al. (2014), Ferreira et al. (2016), Sousa et al. (2016), Sousa et al. (2017), onde foi possível observar similaridade entre as famílias e espécies encontradas.

Foi perceptível uma maior fitodiversidade em outro trabalho desenvolvidos no mesmo bioma, mas com pouca ou sem a presença da invasora, deduzindo-se assim, que o aumento de indivíduos da unha-do-cão pode influenciar na diminuição da biodiversidade local onde se encontram (Sousa et al., 2017).

$\mathrm{O}$ impacto da invasora torna-se mais evidente quando é observada a abundância de indivíduos de $C$. madagascariensis em relação às das demais espécies nativas da região (Tabela 1). De 430 indivíduos inventariados na área, foram registrados que 147 eram da espécie invasora (34\%), mostrando, assim, a capacidade de competição por espaço e recursos da espécie exótica invasora com as demais espécies, dificultando, consequentemente, a permanência de espécies autóctones no ambiente invadido.

De acordo com Fabricante et al. (2015), uma das principais características de espécies invasoras encontradas no bioma caatinga é a formação de densos maciços populacionais por longas extensões. Em campo, foi possível observar os densos maciços de C. madagascariensis, refletindo aos resultados encontrados, elevados valores de índices de dominância, densidade e frequência da espécie. 
Revista Brasileira de Geografia Física V. 12 N. 05 (2019) 1952-1966.

Tabela 1. Parâmetros estruturais da comunidade estudada.

\begin{tabular}{|c|c|c|c|c|c|c|c|c|}
\hline FAMÍLIA/ ESPÉCIE & NOME POPULAR & $\mathrm{N}$ & FA & FR & DA & DR & DoA & DoR \\
\hline \multicolumn{9}{|l|}{ APOCYNACEAE } \\
\hline Cryptostegia madagascariensis Bojer ex Decne. & Unha-do-cão & 147 & 43,21 & 16,83 & 1,81 & 34,19 & 29,25 & 14,86 \\
\hline FAMÍLIA/ESPÉCIE & NOME POPULAR & $\mathrm{N}$ & FA & FR & DA & DR & DoA & DoR \\
\hline \multicolumn{9}{|l|}{ ARECACEAE } \\
\hline \multicolumn{9}{|l|}{ Syagrus cearensis Noblick } \\
\hline Copernicia prunifera (Miller) H. E. Moore & Carnaúba & 90 & 69,14 & 26,92 & 1,11 & 20,93 & 71,25 & 36,19 \\
\hline \multicolumn{9}{|l|}{ BERBERIDACEAE } \\
\hline Berberis laurina Billb. & Espinho-de-judeu & 17 & 13,58 & 5,29 & 0,21 & 3,95 & 3,31 & 1,68 \\
\hline
\end{tabular}

Cochlospermum vitifolium (Willd.) Spreng.

Pacotê

\begin{tabular}{lllllll}
1 & 1,23 & 0,48 & 0,01 & 0,23 & 0,42 & 0,21 \\
\hline
\end{tabular}

\section{CACTACEAE}

\begin{tabular}{lccccccccc}
\hline Cereus jamacaru DC. & Mandacaru & 2 & 2,47 & 0,96 & 0,02 & 0,47 & 0,53 & 0,27 \\
& & & & & & & & \\
& Palma & 1 & 1,23 & 0,48 & 0,01 & 0,23 & 0,6 & 0,31 \\
\hline
\end{tabular}

CAPPARACEAE

\begin{tabular}{|c|c|c|c|c|c|c|c|c|}
\hline Capparis yco (Mart.) & Icó & 2 & 2,47 & 0,96 & 0,02 & 0,47 & 0,47 & 0,24 \\
\hline \multicolumn{9}{|l|}{ EUPHORBIACEAE } \\
\hline Croton blanchetianus Baill. & Marmeleiro-preto & 10 & 7,41 & 2,88 & 0,12 & 2,33 & 2,01 & 1,02 \\
\hline Croton sincorensis Mart. ex Müll. & Marmeleiro-branco & 2 & 2,47 & 0,96 & 0,02 & 0,47 & 0,54 & 0,28 \\
\hline
\end{tabular}

\section{FABACEAE}

Mimosa caesalpiniifolia Benth.

Bauhinia cheilantha (Bong.) Steud.

$\begin{array}{cccccccc}\text { Sabiá } & 51 & 41,98 & 16,35 & 0,63 & 11,86 & 21,65 & 11 \\ \text { Pata-de-vaca } & 1 & 1,23 & 0,48 & 0,01 & 0,23 & 0,15 & 0,08 \\ \text { Unha-de-gato } & 19 & 8,64 & 3,37 & 0,23 & 4,42 & 3,66 & 1,86\end{array}$

Senegalia tenuifolia (L.) Britton \& Rose

Libidibia ferrea (Mart. Ex Tul.) L.P. Queiroz var. Ferrea

\begin{tabular}{cccccccc} 
Jucá & 4 & 4,94 & 1,92 & 0,05 & 0,93 & 0,51 & 0,26 \\
Catingueira & 3 & 1,23 & 0,48 & 0,04 & 0,7 & 0,53 & 0,27 \\
\hline
\end{tabular}

Poincianella bracteosa (Tul.) L.P Queiroz

Catingueira

MALVACEAE

Guazuma ulmifolia Lam.

Mutamba

$74 \quad 50,62$

$19,71 \quad 0,91$

17,21

60,95

30,96

\section{MYRTACEAE}

Campomanesia blanchetiana Landrum \&

M.Ibrahim

$\begin{array}{llllllll}\text { Guabiraba } & 5 & 3,7 & 1,44 & 0,06 & 1,16 & 0,62 & 0,31\end{array}$

N - Número de indivíduos; FA - Frequência absoluta; FR - Frequência relativa; DA - Densidade absoluta; DR - Densidade relativa; DoA - Dominância absoluta; DoR - Dominância relativa.

Barbosa, E. M.; Bonilla, O. H.; Lucena, E. M. P. de; Araújo, L. M. A. de; Oliveira, S. R. S. de. 
Quando comparado os parâmetros estruturais da espécie invasora com a soma dos parâmetros de todas as outras espécies nativas, a espécie exótica apresentou uma densidade relativa e frequência relativa considerável, com valores de 25 e $20 \%$ respectivamente (Figura 2).

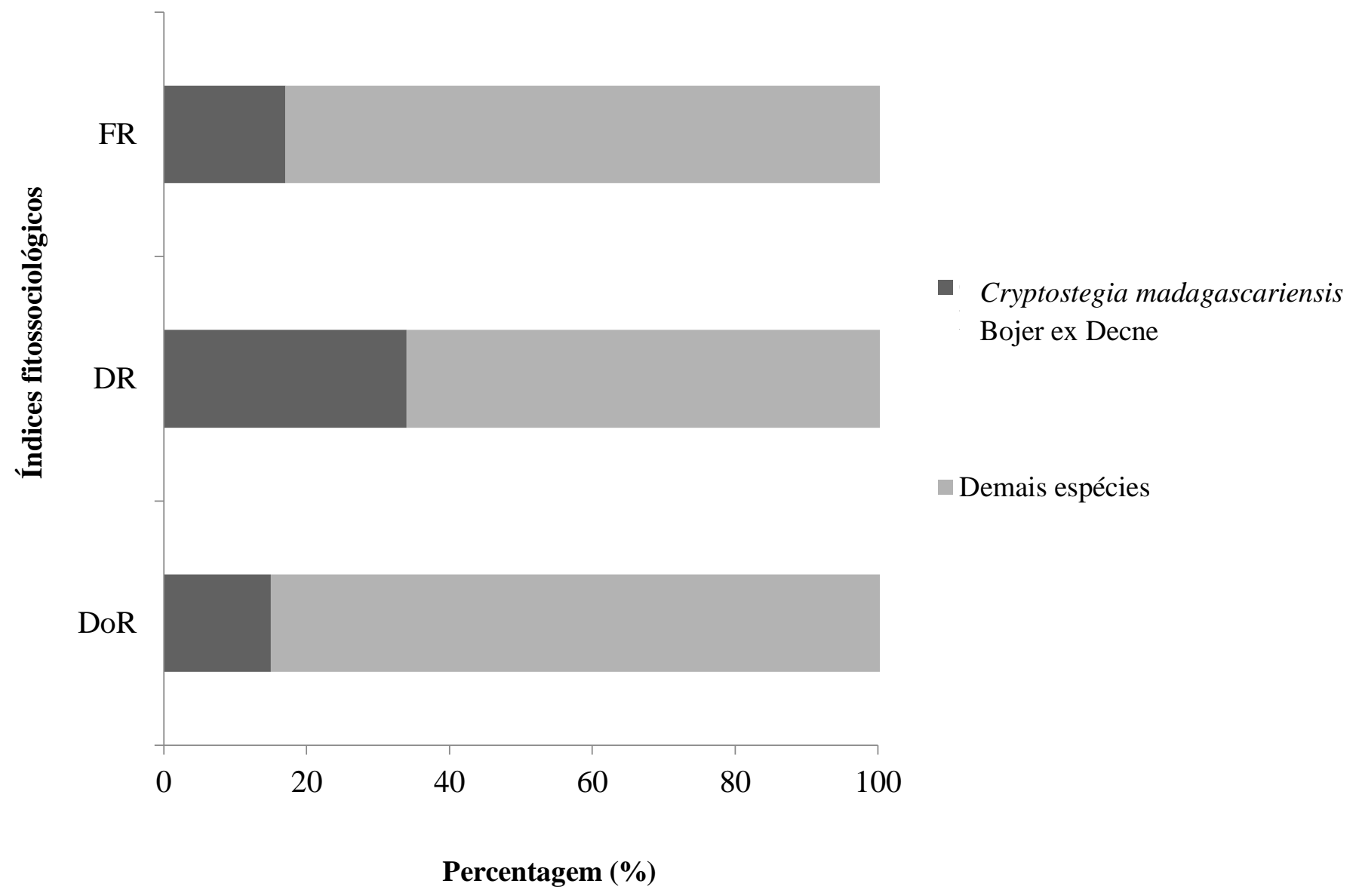

Figura 2. Parâmetros estruturais em percentagem de Cryptostegia madagascariensise Bojer ex Decne. e demais espécies nativas no ambiente avaliado. DoR - Dominância relativa; DR - Densidade relativa; FR Frequência relativa.

O elevado índice de dominância relativa e frequência relativa da espécie invasora $C$. madagascariensis (Figura 3), também foram encontrados no município de Ibaratema, onde valores críticos de 85 e $65 \%$ respectivamente foram apresentados (Sousa et al., 2017).

O elevado número de indivíduos de $C$. madagascariensis pode estar relacionado com diversos fatores morfológicos e fisiológicos da espécie. Essa invasora apresenta elevado número de sementes, podendo chegar a mais de 90 sementes por fruto, além de apresentarem alto índice de germinação, longevidade elevada e capacidade de permanecerem viáveis por longos períodos de seca (Ribeiro et al., 2017). Além disso a sua principal forma de dispersão, por anemocoria, (Figura 3B) é outro fator que potencializa sua proliferação e torna outros ambientes susceptíveis à invasão (Silva et al., 2018).

Assim, constata-se, que apresentam uma alta densidade no banco de sementes da região (Figura 4), sendo um problema ao interferirem no suprimento de novos indivíduos para a comunidade vegetal nativa, dificultando assim uma regeneração natural do ambiente (Schorn et al., 2013). 

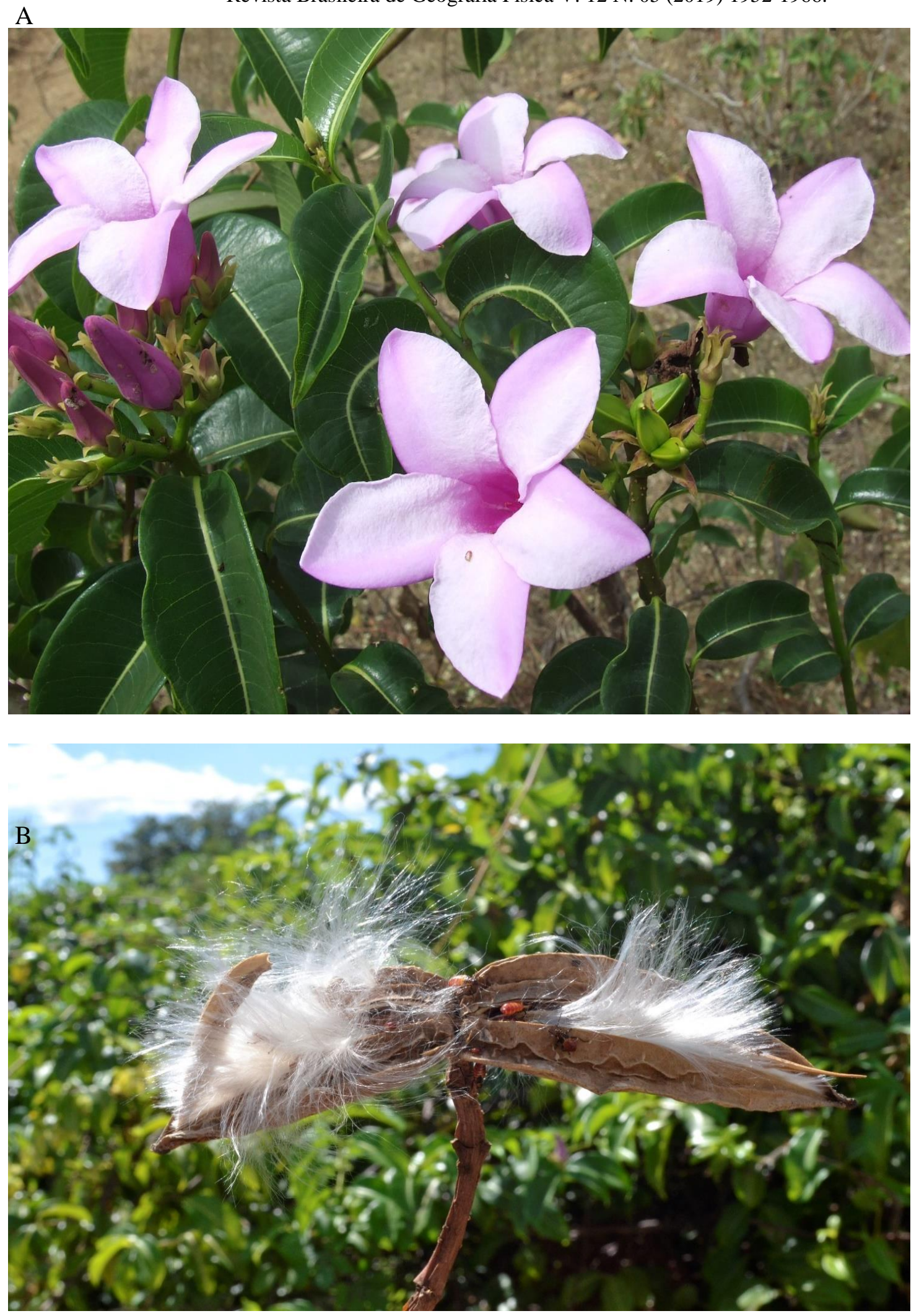

Figura 3. Inflorescência da espécie invasora Cryptostegia madagascariensis Bojer ex Decne (A) e fruto maduro com sementes se dispersando por anemocoria (B). 


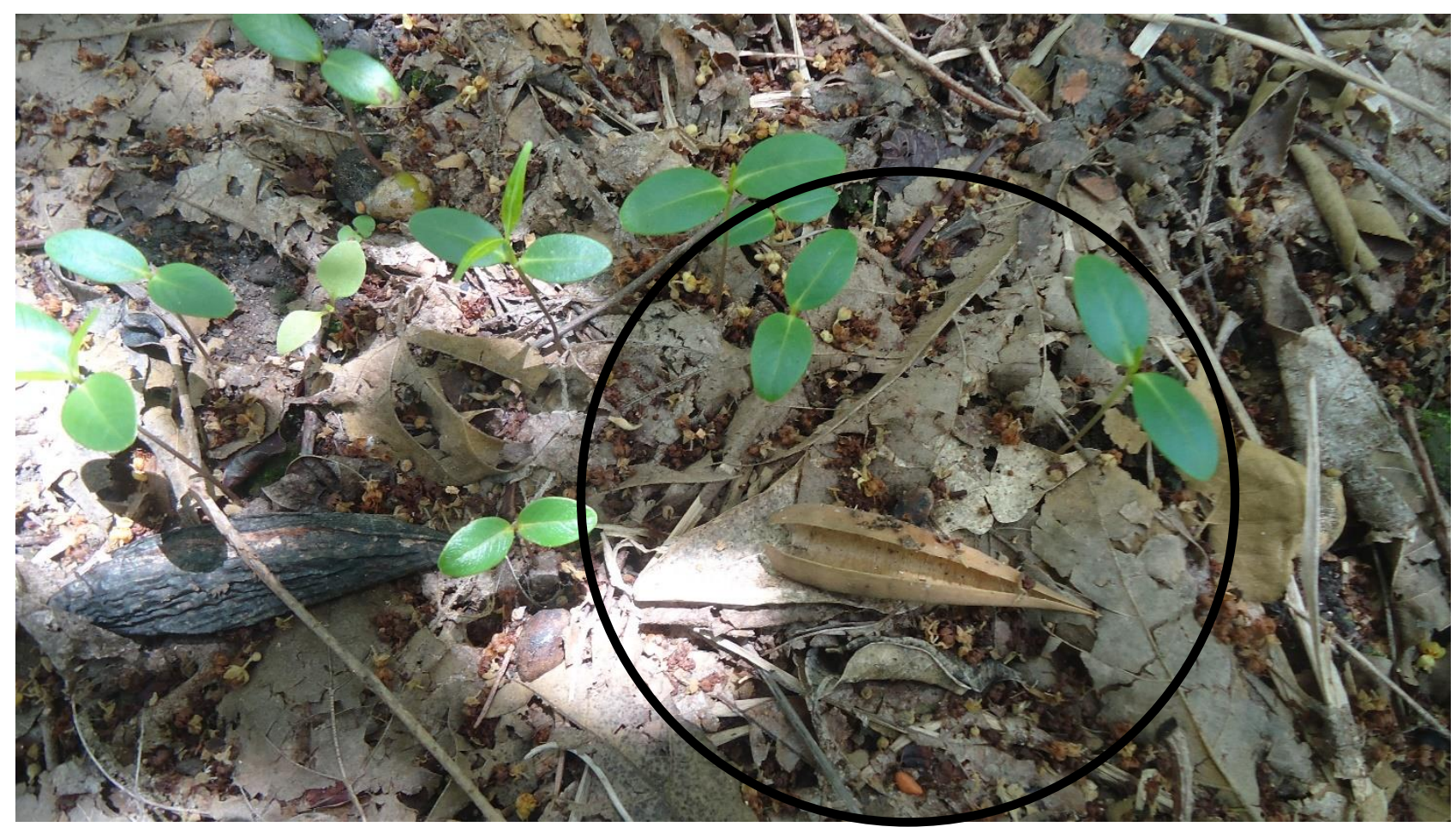

Figura 4. Presença de indivíduos jovens de Cryptostegia madagascariensis Bojer ex Decne, e seu fruto aberto com sementes já dispersas no banco de sementes.

Embora a invasora tenha apresentado altos valores de frequência, a espécie Copernicia prunifera (Mill) H. E. Moore ainda apresentou a maior frequência total da região, porém, muitas palmeiras encontravam-se rodeadas e usadas como suporte pela exótica invasora C. madagascariensis, assim como outras espécies encontradas no mesmo ecossistema, sendo ilustrado na Figura 5 (Vieira et al., 2016).

Em relação ao índice de Shannon (H') os valores de referência são entre 1,5 e 3,5, onde esse último significa que há uma elevada biodiversidade da região analisada. Como resultado tivemos que o a diversidade ( $\left.H^{\prime}\right)$ foi 1,86. Assim podemos caracterizar que o ambiente observado no presente estudo apresentou baixíssima diversidade.

Essa baixa diversidade é comum ser encontrada em ambientes invadidos por espécies exóticas invasoras. No trabalho de Sousa et al (2017) ao avaliarem três ambientes com diferentes níveis de intensidade de invasão pela espécie africana $C$. madagascariensis verificou que nas áreas com maior número dessa invasora a diversidade $\left(H^{\prime}\right)$ local foi bem menor $(0,85)$ do que a diversidade encontrada em áreas com baixa intensidade de invasão $(2,50)$.
Compreender o índice de biodiversidade de um local invadido é fundamental para viabilizar o impacto já causado e desenvolver programas de recuperação dessas áreas (Cabacinha e Lafetá, 2017). Esses resultados que representam a intervenção de invasoras na diversidade local corroboram com estudos que afirmam sobre o principal problema das espécies exóticas quanto à perda global da biodiversidade (Sun et al., 2016).

Populações residentes podem até apresentar espécies em processo de extinção devido à invasividade de espécies exóticas. Essas invasões podem trazer consequências irreparáveis e significativas para o meio. Logo podemos presumir que a presença da espécie $C$. madagascariensis pode ser grande responsável por alterar a fitodiversidade da área estudada, reduzindo consequentemente o valor de importância das espécies nativas inventariadas, uma vez que o valor de importância tende a estar diretamente relacionado com a frequência e densidade relativa da população local (Costa e Mesquita, 2016). 


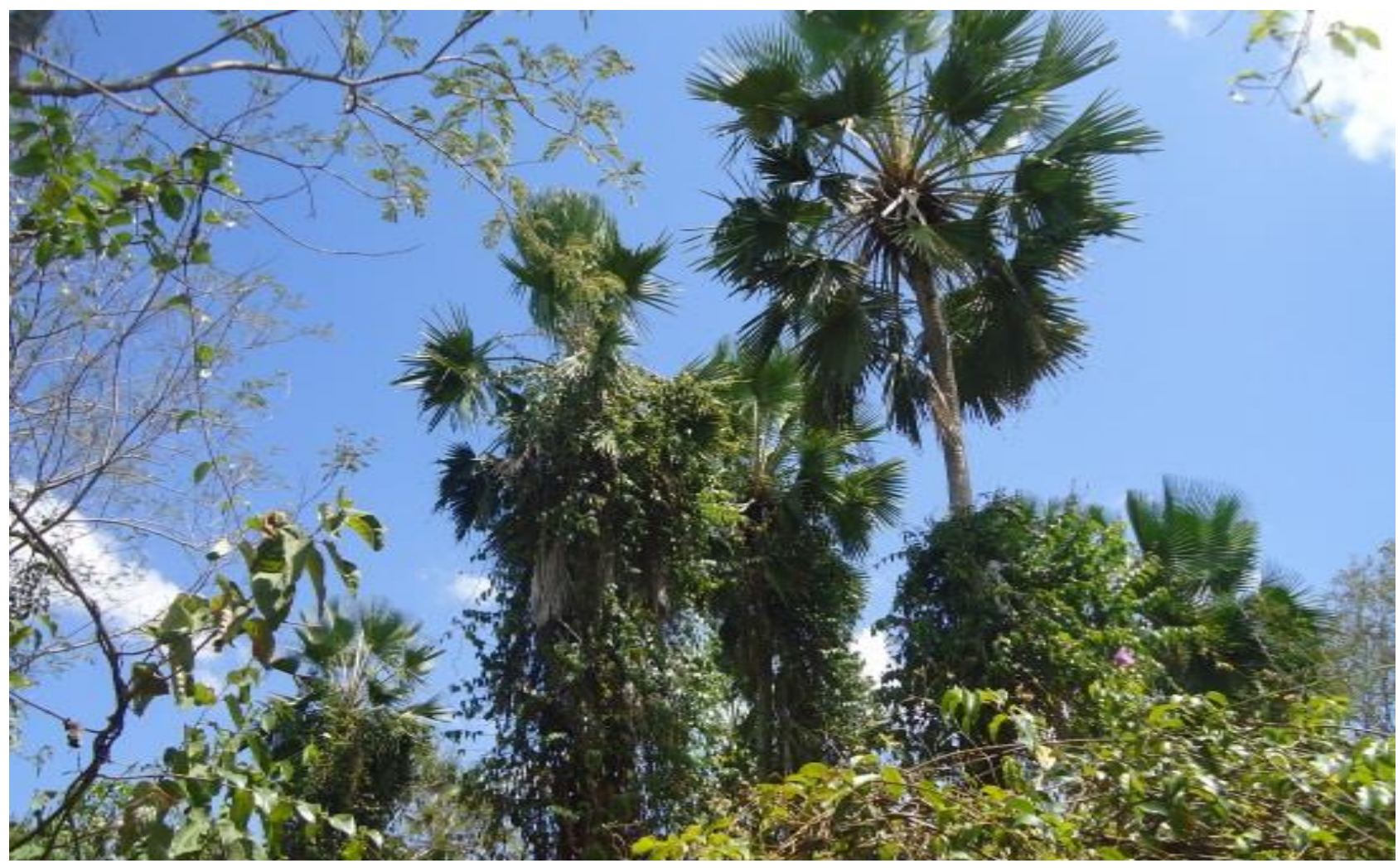

B

$\mathrm{C}$

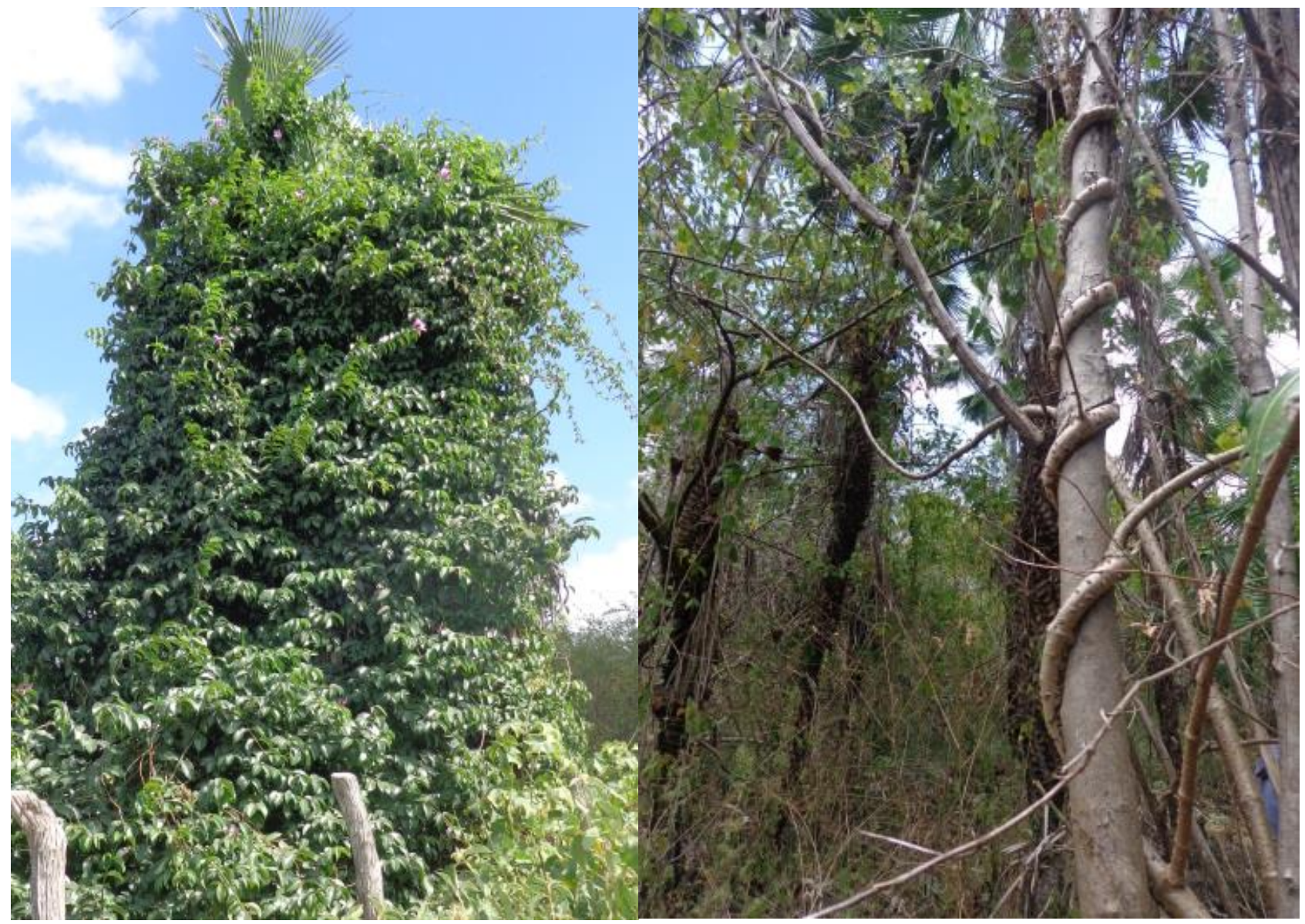

Figura 5. Presença da espécie invasora Cryptostegia madagascariensis Bojer ex Decne e da espécie Copernicia prunifera (Mill) H. E. Moore na área estudada (A). Espécies sendo utilizadas como suporte pela invasora 
africana como, a palmeira Copernicia prunifera (Mill) H. E. Moore (B) e espécies do gênero Croton (C).

A análise estrutural, considerando todas as espécies presentes na área de estudo, apontou $C$. prunifera como a espécie de maior valor de importância VI igual a 33\%, demonstrando assim sua relevância não apenas econômica e social, mas também sua importância ecológica no ecossistema (Figura 6). A segunda com maior VI foi a espécie Guazuma ulmifolia Lam. (mutamba) com VI $27 \%$.
Silva et al. (2016) apresenta que G. ulmifolia é considerada uma espécie secundária inicial, relacionada a estágios iniciais de sucessão.

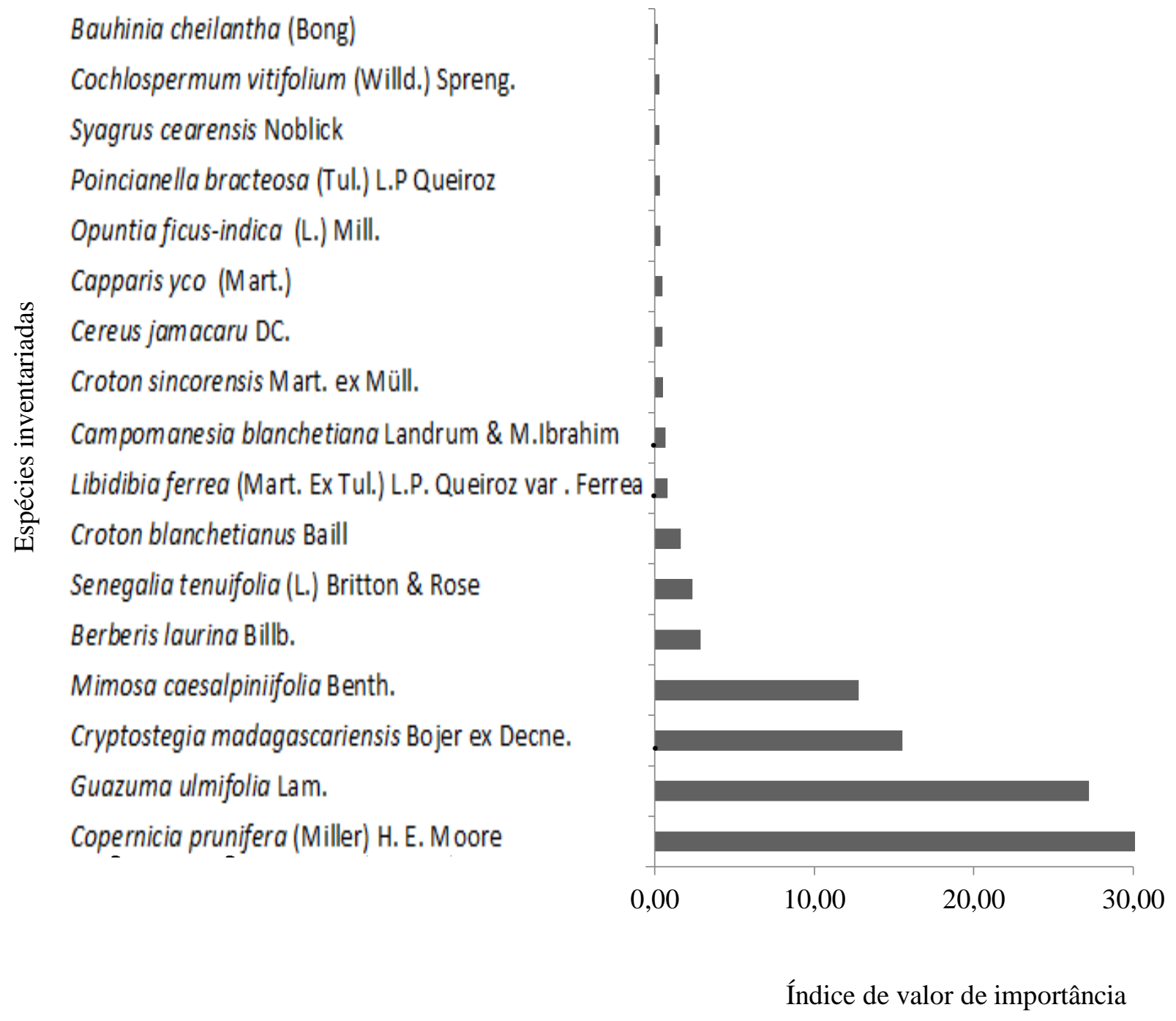

Figura 6. Índice de valor de importância das espécies inventariadas.

O número de indivíduos dessa espécie nativa na área torna-se extremamente importante, visto que há interesse de propagação de espécies nativas para a recomposição da paisagem, seja por invasões ou por outros impactos antrópicos. No entanto quando observamos o valor de importância 
de C. madagascariensis em relação às demais espécies nativas, temos uma discrepância da invasora com as outras 14 espécies constatando assim, mais uma vez, o desequilíbrio estabelecido pela espécie africana no ambiente.

Novamente, a baixa riqueza relacionada com a presença da espécie invasora é ratificada também pelo índice de impacto ambiental de espécies exóticas (IIAE), calculado a partir do coeficiente do impacto ambiental, cujo índice, de acordo com Reaser et al. (2007), variam de -1 a 1, onde quanto mais próximo do extremo negativo, mais acentuado é o grau de invasão, compreendendo que valores negativos indicam que a espécie invasora já causou alterações na estrutura da comunidade.

O valor do IIAE no presente trabalho foi de $-0,69$, onde ainda segundo Reaser et al. (2007), valores abaixo de 0,80 já indicam a ocorrência de danos para a biodiversidade local, pois representam que aproximadamente $20 \%$ da área encontram-se ocupadas apenas pela vegetação invasora, necessitando de intervenções, manejos e monitoramento eficientes no ambiente invadido.

Vale destacar que, o elevado número de espécies invasoras, além de ocasionar danos estruturais nas comunidades invadidas, são responsáveis por causarem alterações nas propriedades ecológicas como no ciclo de nutrientes, produtividade, distribuição de biomassa, acúmulo de serapilheira, mudanças na cadeia alimentar e modificações na relação polinizador planta (Sousa et al., 2017).

Alterações das características químicas e na microbiota do solo são exemplos das modificações que $C$. madagascariensis já vem causando nos ambientes onde invadem. Essas mudanças nas propriedades ambientais que as espécies nativas já se encontravam adaptadas podem ser fator determinante para a diminuição da fitodiversidade, afetando espécies nativas que já se encontravam nessas áreas como a espécie endêmica da caatinga, $C$. prunifera (Souza et al.,2016).

A produção de compostos químicos por $C$. madagascariensis também é outra característica que potencializa sua invasividade. Esses compostos liberados são capazes de inibir a germinação e até desenvolvimento de outras espécies (alelopatia) que, consequentemente, dificultam a realização de uma regeneração natural de um ecossistema (Araújo et al., 2017).

Sabe-se que o índice calculado representa a situação atual da invasão biológica em um determinado ambiente, não sendo capaz de prever futuramente o potencial invasor na comunidade vegetal, fazendo-se necessário uma rápida intervenção no manejo ambiental em áreas que já apresentam a espécie invasora (Santana e Encinas, 2008).

Embora ocorra diversos desafios para o controle e manejo de invasões, devido a constante introdução de espécies exóticas pelas atividades humanas, Halmy et al. (2019) apresenta que já existem técnicas de monitoramento remoto capazes de controlar e evitar invasões, além de possibilitar prever com eficiência as regiões com risco potencial de invasão. Esses monitoramentos também são usados para detectar o nível de degradação dos ecossistemas naturais invadidos (Cardinelli et al., 2017).

Para que ocorra sucesso nos resultados de ações de erradicação de diversas espécies invasoras, entre elas C. madagascariensis, um bom planejamento, persistência e apoio dos órgãos governamentais são imprescindíveis para o êxito.

\section{Agradecimentos}

À Universidade Estadual do Ceará, pela disponibilização do transporte para deslocamento ao local da pesquisa. Aos integrantes do Laboratório de Ecologia da Universidade Estadual do Ceará, pelo apoio na execução das atividades necessárias para a realização do presente trabalho.

\section{Conclusão}

Em função dos resultados obtidos em fragmento de caatinga com presença de Cryptostegia madagascariensis Bojer ex Decne, foi possível concluir que:

1. A espécie exótica invasora Cryptostegia madagascariensis ocasiona impactos estruturais na comunidade vegetal estudada, afetando espécies residentes desde banco de sementes até indivíduos adultos;

2. A espécie invasora apresentou o maior número de indivíduos presentes na área de estudo, com maior frequência, densidade e dominância relativa quando comparada com as demais espécies 
nativas.

3. A palmeira endêmica e de grande importância para a comunidade extrativista, Copernicia prunifera (Mill.) H. E. Moore, é altamente ameaçada pela presença da invasora onde muitas já se encontravam usadas como planta suporte.

4. A espécie invasora diminui a diversidade de indivíduos sendo responsável pela homogeneização de espécies no local.

5. É necessário intervenção urgente no controle e manejo dessa invasora no Estado do Ceará.

\section{Referências}

Almeida, W.R., Lopes, A.V., Tabarelli, M., Leal, I.R., 2015. The alien flora of Brazilian Caatinga: deliberate introductions expand the contingent of potential invaders. Biol Invasions 17, 51-56.

Araújo, H.T.N., Brito, S.F., Pinheiro, C.L., Filho, S.M., 2017. A alelopatia aumenta o potencial invasor de Cryptostegia madagascariensis Bojer ex Decne.? Centro Científico Conhecer 14, 1-12.

Brand, M.A., Oliveira, L.C., Lacerda, S.R., Toniolo, E.R., Júnior, G.L., Campello, R.B., 2015. Caracterização da vegetação da caatinga do Sul do Piauí para geração de energia. Revista Floresta 45, 477-486.

Brito, S.F., Pinheiro, C.L., Nogueira, F.C.B., Filho, S.M., Matos, D.M.S., 2015. Influence of light on the initial growth of invasive Cryptostegia madagascariensis Bojer in the Brazilian semiarid region. Acta Scientiarum. Biological Sciences 37, 385-392.

Byun, C., Blois, S., Brisson, J., 2017. Management of invasive plants through ecological resistance. Biol Invasions 20, 13-27.

Cabacinha, C.D., Lafetá, B.O., 2017. Floristic diversity and equitability in forest fragments using artificial neural networks. Ciência Florestal 27, 143- 152.

Cardinelli, L.S., Fonseca, R.S., Martins, S.V. 2017. Invasão de palmeira australiana no sub-bosque de uma floresta plantada, Viçosa, MG. Pesquisa Florestal Brasileira 37, 1-10.

Costa, J.P., Mesquita, M.L.R., 2016. Floristic and phytosociology of weeds in pastures in Maranhão State, Northeast Brazil. Ciência
Agronômica 47, 414-420.

Costa, V.L.S., Gomes, J.M.A., 2016. Crédito e conservação ambiental no extrativismo da carnaúba (Copernicia prunifera (Mill.) H. E. Moore) no nordeste brasileiro no período de 2007 a 2012. Revista Interações 17, 4-14.

Cruz, F.R.S., Andrade, L.A., Alves, E.U., 2016. Estresse salino na qualidade fisiológica de sementes de Cryptostegia madagascariensis Bojer ex Decne. Ciência Florestal 26, 11891199.

Fabricante, J.R., Araújo, K.C.T., Castro, R.A., Souza, B.S.R., Barros, B.K.R., Filho, J.A.S., 2015. Seleção de espécies autóctones da Caatinga para a recuperação de áreas invadidas por algaroba. Pesquisa Florestal Brasileira 35, $1-10$.

Fabricante, J.R., Castro, R.A., Araújo, K.C.T., Filho, J.A.S., 2015. atributos ecológicos da bioinvasora nicotiana glauca graham (solanaceae) e avaliação da susceptibilidade de sua ocorrência no Brasil. Ciência Florestal 25, 959-957.

Felfili, J.M., Eisenlohr, P.V., Melo, M.M.R.F., Andrade, L.A., Neto, J.A.A.M., 2011. Fitossociologia no Brasil: métodos e estudos de casos, 1. ed. UFV, Viçosa.

Ferreira, R.L.C., Silva, S.O., Silva, J.A.A., Lira, M.A., Júnior, F.T.A., Nascimento, L.M., 2016. Richness and diversity of caatinga areas in different successional stages in northeastern Brazil. Scientia Forestalis 44, 799-810.

Gorgone-Barbosa, E., Pivello, V.R., Rissi, M.N., Zupo, T., Fidelis, A., 2016. A Importância da Consideração de Espécies Invasoras no Manejo Integrado do Fogo. Biodiversidade Brasileira 6, 27- 40.

Halmy, M.W.A., Fawzy, M., Ahmed, D.A., Saeed, N.M., Awad, M.A., 2019. Monitoring and predicting the potential distribution of alien plant species inarid ecosystem using remotelysensed data. Remote Sensing Applications: Society and Environment 13, 69-84.

IPECE. Instituto de Pesquisa e Estratégia Econômica do Ceará, 2009. Perfil básico municipal 2009: Pacatuba, SEPLAG, Fortaleza. Disponível:https://www.ipece.ce.gov.br/wpcontent/uploads/sites/45/2018/09/Pacatuba 2017.pdf. Acesso: 10 jan. 2019.

IPECE. Instituto de Pesquisa e Estratégia Econômica do Ceará, 2017. Perfil básico municipal 2017: Pacatuba, SEPLAG, Fortaleza. Disponível: https://www.ipece.ce.gov.br/wpcontent/uploads/sites/45/2013/01/Pacatuba_Br _office.pdf. Acesso: 10 jan. 2019. 
Johnson, M.D., León, Y.L., 2015. Effect of an Invasive Plant and Moonlight on Rodent Foraging Behavior in a Coastal Dune Ecosystem. Revista Plos One 10, 1-12.

Júnior, L.R.P., Andrade, A.P., Araújo, K.D., Barbosa, A.S., Barbosa, F.M., 2014. Espécies da caatinga como alternativa para o desenvolvimento de novos fitofármacos. Floresta e Ambiente 21, 509-520.

Maitre, D.C., Gush, M.B., Dzikiti, S., 2015. Impacts of invading alien plant species on water flows at stand and catchments scales. AoB Plants 7, 1-21.

Medeiros, J.S., Mesquita, F.O., Andrade, L.A., Oliveira, C.J., Souza, E.M., Souza, J.K.C., 2018. Invasão biológica por Cryptostegia madagascariensis: uma abordagem voltada para estresses abióticos. Pesquisa e Ensino em Ciências Exatas e da Natureza 2, 36-47.

Mueller-dombois, D., Ellenberg, H., 1974. Aims and methods of vegetation ecology. John Wiley and Sons, New York, 547p.

Nackley, L.L., West, A.G., Skowno, A.L., Bond, W.J., 2017. The nebulous ecology of native invasions. Trends in Ecology and Evolution 20, 814-824.

Nascimento, C.E.S., Tarabelli, M., Silva, C.A.D., Leal, I.R., Tavares, W.S., Serrão, J.E., Zanuncio, J.C., 2014. The introduced tree Prosopis juliflora is a serious threat to native species of the Brazilian Caatinga vegetation. Science of the Total Environment 481, 108-113.

Nobis, A., Nowak, A., Rola, K., 2018. Do invasive alien plants really threaten river bank vegetation? A case study based on plant communities typical for Chenopodium ficifolium- An indicator of large river valleys. Plos One 13, 1-15.

Padalia, H., Srivastava, V., Kuswaha, S.P.S., 2015. How climate change migt influence the potential distribution of weed, bushmint (Hyptis suaveolens)? Enviromental Monitoring and Assessment 187, 1-14.

Pereira, D.S., Sousa, J.E.S., Pereira, M.S., Gonçalves, N.R., Bezerra, A.M.E., 2014. Emergence and initial growth of Copernicia prunifera (Arecaceae) as a function of fruit maturation. Journal of Seed Science 36, 09-014.

Reaser, J.K., Meyerson, L.A., Cronk, Q., Poorter, M., Eldrege, L.G., Green, E., Kairo, M., Latasi, P., Mack, R.N., Mauremootoo, J., O’Dowd, D., Opara, W., Sastroutomo, S., Saunders, A., Shine, C., Thrainsson, S., Vaiutu, L., 2007.
Ecological and socioeconomic impacts of invasive alien species in island ecosystems. Environmental Conservation 34, 98-111.

Ribeiro, T.O., Bakke, I.A., Souto, P.C., Bakke, O.A., Lucena, D.S., 2017. Diversidade do banco de sementes em diferentes áreas de caatinga manejadas no semiárido da Paraíba, Brasil. Ciência Florestal 27, 203-213.

Rocha, T.G.F., Silva, R.A.R., Dantas, E.X., Vieira, F.A., 2015. Fenologia da Copernicia prunifera (Arecaceae) em uma área de caatinga do Rio Grande do Norte. Cerne 21, 673-682.

Rumlévora, Z., Vilá, M., Pergl, J., Nentwig, W., Pysek, P., 2016. Scoring environmental and socioeconomic impacts of alien plants invasive Europe. Biol Invasions 18, 3697-3711.

Santana, O.A., Encinas, J.I., 2008. Levantamento das espécies exóticas arbóreas e seu impacto nas espécies nativas em áreas adjacentes a depósitos de resíduos domiciliares. Biotemas 21, 29-38.

Schorn, L.A., Fenilli, T.A.B., Krüger, A., Pellens, G.C., Budag, J.J., 2013. Composição do banco de sementes no solo em áreas de preservação permanente sob diferentes tipos de cobertura. Revista Floresta 43, 49-58.

Shannon, C.E., Weaver, W., 1949. The mathematical theory of communication. Urbana, IL: University of Illinois Press.

Silva, D.L.S., Luz, G.R., Veloso, M.D.M., Fernandes, G.W., Nunes, Y.R.F., 2016. Emergência e estabelecimento de plântulas de Guazuma ulmifolia Lam. em função de diferentes tratamentos pré-germinativos. Ciência Florestal 26, 763-772.

Silva, L.G.C., Moreira, J.F.L., Holanda, H.B.B., Rocha, E.L.B., Dias, P.C., 2018. Evaluation of carnauba progenies and estimates of genetic parameters in the juvenile phase. Revista Caatinga 31, 917- 925.

Silva, S.F., Costa, H.S.L., Viana, J.S., Filho, S.M. 2018. Fitotoxidez de espécies exóticas sobre o potencial fisiológico de sementes de crambe (Crambe abyssinica Hochs). Revista Agroambiente 12, 89-95.

Skálová, H., Moravcová, L., Dixon, A.F.G., Kindlmann, P., Pysek, P., 2015. Effect of temperature and nutrients on the growth and development of seedlings of an invasive plant. AoB Plants 7, 1-8.

Sousa, F.Q., Andrade, L.A., Xavier, K.R.F., 2016. Cryptostegia madagascariensis Bojer ex Decne: impactos sobre a regeneração natural em fragmentos de caatinga. Revista Brasileira de 
Ciências Agrárias 11, 39-45.

Sousa, F.Q., Andrade, L.A., Xavier, K.R.F., Silva, P.C.C., Albuquerque, M.B., 2017. Impactos da invasão de Cryptostegia madagascariensis Bojer ex Decne. (Apocynaceae Juss.) em remanescentes de caatinga no município de Ibaretama, Ceará, Brasil. Ciência Florestal 27, 1243-1255.

Sousa, R.F., Silva, R.A.R., Rocha, T.G.F., Santana, J.A.S., Vieira, F.A., 2015. Etnoecologia e etnobotânica da palmeira carnaúba no semiárido brasileiro. Cerne 21, 587-594.

Souza, T.A.F., Rodriguez-Echeverría, S., Andrade, L.A., Freitas, H., 2016. Could biological invasion by Cryptostegia madagascariensis alter the composition of the arbuscular mycorrhizal fungal community in semi-arid Brazil? Acta Botanica Brasilica 30, 93-101.

Souza, V.C., Andrade, L.A., Quirino, Z.G.M., 2016. Floral biology of Sesbania virgata: an invasive species in the Agreste of Paraíba, northeastern Brazi. Rodriguésia 67, 871-878.

Sun, Y., Müller- Schärer, H., Schaffner, U., 2016. Neighbour origin and ploidy level drive impact of an alien invasive plant species in a competitive environment. Plos One 11, 1-14.

Vieira, I.R., Oliveira, J.S., Loiola, M.I.B., 2016. Effects of harvesting on leaf production and reproductive performance of Copernicia prunifera (Mill.) H. E. Moore. Revista Àrvore 40, 117-123. 